\title{
Las TIC como auxiliares lingüístico-didácticos en educación indígena
}

\section{ICT as linguistic-didactic aids for indigenous education}

\author{
Salvador Galindo-Llaguno ${ }^{1}$ \\ Fecha de recepción: 21 de marzo de 2019 \\ Fecha de aceptación: 25 de julio de 2019
}

\begin{abstract}
Resumen - Ante la falta de insumos propios y materiales de intervención didáctica desarrollados mediante las Tecnologías de la Información y la Comunicación (TIC), se presenta este artículo en el que se describen, grosso modo, los aciertos y desaciertos de la atención pedagógica que han recibido los grupos indígenas de México a través de diversos proyectos e instituciones, así como de la Secretaría de Educación Pública (SEP). Posteriormente, se refiere al uso de las TIC como auxiliares de aprendizaje para una educación intercultural y bilingüe mediante el uso de dos tipos de software educativos y una aplicación móvil, productos de un proceso emergente de investigación y documentación lingüística con la participación de un grupo multidisciplinario de profesionales y hablantes nativos de las diversas lenguas del estado de Oaxaca, México.

Por último, a manera de reflexión, se expone una serie de recomendaciones para el diseño e inserción de las TIC en los planes y programas de estudio de la modalidad de educación indígena en México y la insistencia de producir materiales educativos multimedia con enfoques de diversidad lingüística y cultural.
\end{abstract}

\section{Palabras clave:}

TIC, educación indígena, educación bilingüe-intercultural, diversidad lingüística.
Abstract - This paper roughly describes the achievements and failures in pedagogical attention that the Mexican indigenous groups have received through several projects and institutions, as well as the Secretariat of Public Education, facing the lack of their own didactic supplies and didactic intervention materials developed through Information and Communication Technologies (ICT). Afterward, ICT are presented as a learning tool to achieve an intercultural and bilingual education through the use of two types of educational software and a mobile application, as a result of an emerging process of linguistic research and documentation with the participation of a multidisciplinary group of professionals and native speakers of the different languages from the state of Oaxaca, Mexico.

Lastly, as something worth considering, a series of recommendations for the design and insertion of ICT in the plans and programs of study of the modality of indigenous education in Mexico is presented, along with the urging of producing multimedia educational materials with linguistic and cultural diversity approaches.

\section{Keywords:}

ICT, indigenous education, bilingual-Intercultural education, and linguistic diversity. 


\section{Introducción}

1 no de los grandes retos que enfrenta el personal docente de educación indígena de México es la escasez de materiales educativos multimedia que respondan de manera oportuna e idónea a las necesidades de niñas y niños hablantes de una lengua indígena. Tampoco podemos dejar de considerar la falta de innovación tecnológica por parte de maestras y maestros para subsanar de alguna forma dicho desafío.

Respecto a la responsabilidad del magisterio sobre su quehacer docente y sus incidencias en el estudiantado se ha hablado mucho; han surgido diferentes acciones o teorías que lo orientan para repensar constantemente lo que se está haciendo y sobre cómo mejorar su práctica educativa, con la finalidad de lograr un proceso abierto y constante de una profesionalización crítica que lo conduzca a realizar operaciones reflexivas e innovadoras que transformen su contexto escolar y comunitario. Por el contrario, en relación con el proceso de diseño y producción de materiales multimedia novedosos para ambientes bilingües interculturales se ha escrito muy poco y, por ende, dicha situación se ha ido rezagando o descontextualizando.

Por lo anterior, partiendo de una descripción histórica de los proyectos e instituciones para la instrumentación de una educación intercultural bilingüe, y ante la falta de materiales e insumos propios para atender el ámbito escolar indígena, el presente trabajo tiene por objetivo contribuir y promover la explicación de diversos materiales interactivos y aplicaciones digitales con pertenencia cultural y lingüística del estado de Oaxaca, México, así como subrayar la importancia de continuar en el desarrollo y diseño de aplicaciones y materiales educativos multimedia adecuados para contextos de diversidad lingüística y cultural. Además, pretende demostrar que sí es posible innovar en el quehacer cotidiano dentro del aula, aprovechando todo tipo de insumos, instrumentos y materiales tecnológicos que se encuentren a nuestro alrededor.

\section{Aciertos y desaciertos en la atención pedagógica de los grupos indígenas}

Desde la creación de la Secretaría de Educación Pública (SEP), en 1921, hasta la actualidad, la idea de consolidar el Estado-nación y estructurar un proyecto educativo para lograr el desarrollo del país no ha permitido a las comunidades originarias ninguna posibilidad de trascender, puesto que su condición de marginalidad y explotación, sumado a la discriminación social y racial que padecen, aún son evidentes, negándoles toda oportunidad de progreso a su lengua y cultura.

Los proyectos e instituciones que se conformaron posteriormente a la creación de la SEP, así como los acontecimientos que en apariencia favorecían el uso de la lengua originaria -pero que en realidad eran el vínculo de castellanización y alfabetización de los grupos étnicos- fueron la Estación Experimental de Incorporación Indígena de Carapan en 1932, los 11 internados indígenas en el mismo año, el establecimiento del Instituto Lingüístico de Verano en 1935, el Departamento de Asuntos Indígenas en 1936, el Proyecto Tarasco en 1939, el Primer Congreso Indigenista Interamericano en 1940, el Instituto de Alfabetización en Lenguas Indígenas en 1944, la Dirección General de Asuntos Indígenas en 1946, el Instituto Nacional Indigenista en 1948. Asimismo, el Servicio Nacional de Promotores Culturales y Maestros Bilingües en 1963, la Dirección General de Educación Extraescolar en el Medio Indígena en 1971, la Coordinación General de los Servicios Educativos para Zonas Deprimidas y Grupos Marginados en 1977, y la Dirección General de Educación a Grupos Marginados y la Dirección General de Educación Indígena, ambos en 1978, esta última encargada de hacer realidad en el medio indígena el Plan Nacional de Educación para Todos, cuyos programas prioritarios iniciaron con la castellanización primaria para toda la infancia y la educación de personas adultas.

En relación con los proyectos e instituciones antes enlistados, dentro de sus políticas y en el diseño de 
sus planes y/o estrategias en ningún momento han contemplado de manera enérgica el desarrollo de las lenguas y culturas propias de los pueblos originarios. Apenas hablan de conservarlas, pero no explican las estrategias ni los procedimientos que deben seguirse para hacer efectivo este postulado y, más aún, no se garantizan legalmente los recursos y mecanismos para evitar que las lenguas y culturas originarias no resulten afectadas o, en el peor de los casos, se extingan. Dicho criterio no significa que no se reconozca la voluntad política actual hacia los pueblos originarios, puesto que ya existen leyes y tratados nacionales e internacionales que priorizan el uso y la permanencia de las lenguas y culturas originarias.

Con todo, dentro de las políticas de los planes nacionales de desarrollo y de los proyectos educativos implementados por la Dirección General de Educación Indígena, "no puede hablarse de una educación indígena, si la filosofía, los objetivos, los contenidos de planes y programas, la metodología y la forma de evaluación son de la cultura occidental, o dominante, sin que la cultura indígena se haga presente" (Hernández, 1979, p. 51). Para tener presencia dentro del ámbito educativo $y$, en general, a nivel nacional, es necesario elaborar diversos materiales que fortalezcan las lenguas originarias, pero, sobre todo, que las instituciones y los distintos organismos gubernamentales y no gubernamentales implementen planes y programas de estudio con contenido intercultural y valores filosóficos de las culturas originarias que aún prevalecen en nuestra nación.

Respecto a los auxiliares didácticos destinados a la población indígena, se ha observado que éstos han sido generados por diversas instituciones $u$ organismos ajenos al contexto comunitario indígena, por lo que se tienen que adaptar y reformular para su aplicación en entornos escolares distantes a aquéllos donde fueron creados y en realidad sólo resultan ser una enmienda provisional. Sobre esto, Nolasco precisa:
Si a eso agregamos otros aspectos, como el material didáctico, que es escaso e inapropiado, se tendrá el panorama del ineficiente sistema escolar que solo produce fracasos educativos. Como meros ejemplos del material didáctico podrían mencionarse las cartillas bilingües, que carecen ya no de un método sino hasta de lógica y de sentido común, y que manejan los idiomas (español y/o lenguas indígenas) con un completo desconocimiento de las estructuras de los mismos, y, con frecuencia, usando como base analógica al inglés (Nolasco, 1978, p. 255).

Dicha situación también se puso de manifiesto desde el I Congreso Nacional "Los indígenas y su política educativa", organizado por la Alianza Nacional de Profesionales Indígenas Bilingües A. C. (ANPIBAC), cuyos participantes revelaron:
Haciendo un análisis crítico en materia de educación en el medio indígena, honestamente hablando, lo que hemos hecho los promotores y maestros bilingües, es llevar a la práctica sistemas educativos totalmente incongruentes para los indígenas, ya que todo lo planeado y programado, ha sido por gentes mestizas y por tanto desconocedoras del medio socio-cultural y lingüístico de los indígenas (ANPIBAC, 1980).

A la luz de la evidencia anterior, en el caso de nuestro país, el Plan Nacional para la Instrumentación de la Educación Indígena Bilingüe-Bicultural, propuesto por la ANPIBAC al gobierno de México alrededor de 1980 , propicia y exige la participación de los pueblos originarios en la investigación, elaboración y evaluación de planes y programas, así como los 
contenidos y metodologías de la educación indígena en cada grupo étnico.

Por un lado, para realizar el proceso de instrumentación, establecer metodologías y generar materiales didácticos acordes con la cosmovisión indígena, se conforma un equipo de maestras y maestros bilingües bajo la coordinación técnica de profesionales de la Lingüística y de la Pedagogía, quienes en forma conjunta empiezan la elaboración de auxiliares didácticos, manuales y gramáticas de las lenguas indígenas como mecanismos de transmisión de la cultura a las nuevas generaciones. Por otro lado, el Estado implementa el diseño de diversos auxiliares didácticos, pero con una política de integración nacional, lo cual López y Rivas critica así:

Ante esta política de integración económica y territorial, de imposición lingüística y cultural, los grupos que difieren en su composición étnica de la nacionalidad dominante en formación, se ven sujetos, entre otros, a un proceso de desintegración cultural que paulatina o rápidamente (según sean las circunstancias concretas), los lleva a un estado de anomia, a la pérdida de la identidad étnica, y a la desaparición cultural del grupo y a su "incorporación" a los sectores más explotados de la nacionalidad dominante (López y Rivas, 1978, p. 38).

Entre los materiales y auxiliares didácticos producto de las coordinaciones técnicas bilingües y del Estado podemos encontrar desde Las cartillas bilingües (Escalante, 2013), una compilación de narraciones en lengua indígena, perspectivas de ortografías fonémicas de lenguas indígenas, alfabetos prácticos y consolidados en lengua originaria hasta la edición, por parte de la Secretaría de Educación Pública (SEP) a través del Programa de Educación Preescolar Bilingüe y Primaria Bilingüe Bicultural para todos los niños indígenas (SEP-
CONAFE-INI, 1981), hasta tres apoyos didácticos detallados a continuación:

a) Mi primer libro, cuadernillo con ilustraciones a color, sin textos, que contenían ejercicios de maduración para la lectoescritura.

b) Mi libro de lectoescritura en lengua indígena, que se elaboró en 35 lenguas y más de 70 variantes dialectales.

c) Lectoescritura en lengua indígena.

d) Libro del maestro, consistente en una guía lingüística y didáctica complementaria al texto Mi primer libro.

Estos materiales fueron estructurados a manera de texto y están desfasados contextualmente, por no considerar diversos aspectos de pertenencia comunitaria; lingüísticamente, por no atender la variación dialectal existente; y didácticamente, por no migrarlos a los beneficios técnico-didácticos digitales actuales ni a las plataformas con herramientas educativas que se ofrecen al resto de la población nacional no indígena. Esta situación también se enmarca y se hace evidente en otras investigaciones, tal como se muestra en el artículo "Educación, interculturalidad y tecnologías de la información y la comunicación.Hacia la construcción de un estado del arte en México", donde en una de sus conclusiones puntualizan:

\begin{abstract}
En la literatura revisada hasta este momento no existe un antecedente directo que conjugue la relación de los medios y TIC con la educación intercultural, menos aún, con un marco teórico metodológico que rescate las aportaciones de las ciencias de la comunicación (Ortiz, Hernández \& Romero, 2014, p. 17).
\end{abstract}

Sin menoscabo de lo anterior, se aclara que dichos recursos representan un gran progreso en la atención pedagógica de los grupos indígenas. 
Uso de las TIC en auxiliares de aprendizaje para una educación intercultural y bilingüe

El empleo de las TIC en las aulas de educación primaria se ha dado de manera paulatina y ya no es algo nuevo, puesto que el mundo en el que vivimos se encuentra en un continuo proceso de cambio, lo cual provoca inéditas formas de relación e interacción entre los medios y los seres humanos. Así surgen distintas corrientes de pensamiento pedagógico, métodos novedosos y, sobre todo, nuevos auxiliares didácticos de enseñanzaaprendizaje. Frente a esta realidad, Guerrero y Dote (2011) enfatizan que en el actual contexto educativo pareciera que una de las demandas más fehacientes por parte de las políticas públicas de todos los países del mundo es la apropiación de todo tipo de recursos tecnológicos para lograr el mejoramiento en la calidad y equidad de los centros educativos. Es así como las TIC nos ofrecen un sinnúmero de posibilidades en nuestras prácticas educativas, entre las que destacan: la ampliación de la oferta informativa, creación de entornos más flexibles para el aprendizaje, eliminación de las barreras espacio-temporales, potenciación de escenarios y de entornos interactivos, entre otras posibilidades.

Por ello, las metodologías didácticas que se han de emplear en los centros educativos deben aproximarse a las transformaciones sociales contemporáneas para así contribuir con aportes cognitivos o críticas de pensamiento entre el alumnado, con el apoyo y acceso de las TIC. Por ejemplo, en décadas pasadas el gobierno mexicano instrumentó la radio como proceso de enseñanza-aprendizaje mediante el programa radiofónico bilingüe del cual Hernández describe: "Desde 1958 se comprobó, a través del sistema de Escuelas Radiofónicas Bilingües de la Mixteca Alta Oaxaqueña, la eficacia de la radio como apoyo en la dirección del proceso de enseñanza-aprendizaje de tipo normal" (Hernández, 1982, p. 128).

Posteriormente, en el sexenio de Gustavo Díaz Ordaz (1964-1970), con Agustín Yáñez al frente de la SEP se instauró el uso televisivo como medio educativo formal, donde:

El 17 de agosto de 1965 se anunciaron por primera vez en el periódico Novedades, el proyecto de Alfabetización y el de Educación Secundaria por televisión, mencionando que en algunos países el uso de este medio había generado mejores resultados educativos y en menor tiempo que los métodos tradicionales (Jiménez, 2010, p. 25).

En 1995, el gobierno federal, mediante la SEP, suscribe un convenio de cooperación con el Instituto Latinoamericano de Comunicación Educativa (ILCE) para coadyuvar en la operación de la red Edusat con programas de educación a distancia y el uso de las TIC, así como de educación no formal para la capacitación de servidoras y servidores públicos o de actualización docente. Con estos ejemplos, enfatizamos que se han incluido en el ámbito educativo acorde con los adelantos tecnológicos de cada era. Por lo tanto, ¿por qué no hacer uso de las innovaciones tecnológicas actuales en el contexto educativo indígena? Al respecto, hay quienes opinan que la falta de implementación de las TIC en contextos indígenas incrementa aún más su condición de marginación. Según el primer taller indígena de este tipo de tecnologías digitales, auspiciado por la International Telecommunication Union y la Comisión Nacional para el Desarrollo de los Pueblos Indígenas, realizado en México del 28 al 30 de noviembre de 2005:

[su uso] se ha convertido en una nueva forma de exclusión de los indígenas, al mismo tiempo que, por un lado ayudan a lograr una democratización en el uso de distintos recursos $y$, por otro, innumerables posibilidades de crecimiento a los indígenas, que, por 
diversas razones en su mayoría, son marginados del ciberespacio. Hablamos de una nueva forma de exclusión social y política, con centralización del poder que basa sus mecanismos en un injusto acaparamiento de los recursos tecnológicos por parte de intereses políticos y mercantilistas (Sandoval \& Mota, 2007, s.p.).

Ante tal panorama, y con la visión de aprovechar las TIC en contextos indígenas, se deben generar materiales educativos multimedia que se concreten en el desarrollo y la producción de programas y actividades virtuales interactivas grabadas en soporte digital bilingüe o multilingüe, los cuales coadyuven a impartir una educación intercultural y bilingüe de manera alternativa; además de apoyar en el fortalecimiento, desarrollo y preservación de las lenguas indígenas y en la promoción de los valores lingüísticos, culturales y comunitarios.

\section{Auxiliares lingüístico-didácticos soportes 0 entornos digitales}

El tipo de materiales que nos ocupa debe promover procesos de observación, experimentación y conocimiento sobre aspectos culturales y lingüísticos que sirvan como ejes transversales destinados a apoyar los procesos educativos que favorezcan la reflexión en los usuarios sobre la interculturalidad y su derecho a pertenecer a un determinado grupo étnico. De antemano, habrá que reconocer la existencia de muchas limitantes para que las escuelas situadas en comunidades indígenas o rurales reciban los beneficios potenciales que ofrecen las TIC. No obstante, es preciso considerar que dichas tecnologías avanzan de manera vertiginosa y cada vez más se tornan en herramientas imprescindibles en la vida cotidiana de las personas y se posicionan rápidamente en cualquier espacio geográfico.
Frente a esta situación, el Centro de Estudios y Desarrollo de las Lenguas Indígenas de Oaxaca (CEDELIO) está incursionando en el diseño e implementación de nuevos auxiliares de aprendizaje mediante el uso de las TIC, con una pertinencia indígena basada en la lengua originaria y con una participación cultural incluyente de otras culturas, en el afán de generar una conciencia lingüística.

EI CEDELIO, mediante un proceso de investigación y documentación lingüística y con la participación de un grupo multidisciplinario de profesionales del Instituto Nacional de Lenguas Indígenas (INALI), del Centro Profesional Indígena de Asesoría, Defensa y Traducción, A. C. (CEPIADET, A. C.) y de la Corporación Oaxaqueña de Radio y Televisión (CORTV), ha incursionado en el diseño e implementación de un software lingüístico-educativo denominado Xjuaní: vocabulario ilustrado e interactivo en nueve lenguas indígenas de Oaxaca, en el cual intervinieron hablantes nativos del huave, mixe, chatino, chinanteco, cuicateco, mazateco, ixcateco, chocholteco y mixteco.

Además, se contó con la colaboración de profesionales del diseño gráfico, programación informática, lingüistas y docentes, quienes, de manera conjunta, sugirieron estrategias y secuencias didácticas que facilitaran la comprensión de los contenidos o ejes temáticos que abarcan las diferentes asignaturas del esquema básico de educación. Se conformó así un programa interactivo que comprende cinco campos semánticos: cuerpo humano, casa-hogar, flora, fauna y parentesco.

Como bien señalan Villavicencio y Salgado (2011, p. 12): "Para garantizar un mejor desarrollo de los programas, será imprescindible la participación de especialistas en educación, capaces de garantizar que los programas estén adecuadamente diseñados y cumplan de manera óptima los objetivos que persigue cada uno".

Al tomar en cuenta lo anterior, la concepción y confección del software lingüístico-educativo Xjuaní 
promovió procesos de observación, experimentación y conocimiento sobre aspectos culturales y lingüísticos, los cuales sirvieron como ejes transversales destinados a apoyar los procesos educativos que susciten la reflexión de usuarias y usuarios sobre la interculturalidad y sobre sus derechos a pertenecer a un determinado grupo étnico.

Posteriormente, con base en el conocimiento adquirido con Xjuaní, de manera interinstitucional y colaborativa con alumnos de la Ingeniería en Tecnologías de la Información y Comunicación de la Universidad Tecnológica de los Valles Centrales de Oaxaca (UTVCO), y considerando que los procesos digitales han ganado mayor presencia en dispositivos móviles (smartphones), se procedió al diseño y desarrollo de la aplicación (app) Yalam cuyo objetivo es concientizar y sensibilizar a los usuarios sobre la riqueza lingüística del estado de Oaxaca; además, mediante el uso de las nuevas tecnologías como teléfonos móviles y tabletas electrónicas se permite a los interesados interactuar de forma fácil y didáctica con algunas palabras de las variantes dialectales existentes de las 16 lenguas originarias que aún prevalecen en Oaxaca.

A partir de las experiencias descritas conanterioridad, y mediante un sentido de corresponsabilidad para que la población infantil y juvenil de la comunidad de Santa María del Tule, Oaxaca, se sensibilice, aprenda y preserve su lengua originaria, los adultos mayores hablantes del idioma zapoteco del Tule, en coordinación con el CEDELIO y la Dirección de Tecnologías Educativas del Instituto Estatal de Educación Pública de Oaxaca (IEEPO), sumaron esfuerzos para desarrollar Yagiits, un software didáctico interactivo bilingüe (zapoteco-español) con enfoque cultural que permite fortalecer la identidad y despertar la sensibilidad lingüística de hablantes y no hablantes del zapoteco, que se desarrolló con la participación directa de los habitantes de esa comunidad, quienes aportaron los elementos lingüísticos y culturales del zapoteco del Tule, aprovechando así las oportunidades que ofrecen las TIC.

\section{Conclusión}

Como resultado del diseño de materiales interactivos con pertinencia cultural y lingüística, se reflexionó sobre una serie de recomendaciones para el desarrollo e inserción de las TIC en los programas educativos de la modalidad de educación indígena, donde se consideraron los siguientes puntos:

1. Los programas multimedia deben responder a los contextos reales de uso.

2. Es preciso analizar las principales características que deben presentar los programas para su posterior uso en el aula.

3. Deben ser flexibles y con una interfaz amigable.

4. Deben ser recursos de aprendizaje para la convivencia y la paz, inculcando valores y principios sociales necesarios para convivir en armonía.

5. Deben promover un aprendizaje colaborativo, que permita al alumnado trabajar en equipo.

6. Es importante motivar al estudiantado con respecto a la información y los contenidos educativos mediante su lengua materna.

7. Tienen que favorecer un aprendizaje significativo que posibilite la reestructuración de conocimientos previos y la construcción de otros nuevos.

8. Que promuevan un bilingüismo coordinado, es decir, que el alumnado utilice ambas lenguas de manera independiente y adecuada según la situación.

9. Que planteen procesos de evaluación y autoevaluación.

10. Que tengan una función formativa global para el análisis y construcción de nuevos conocimientos.

Por lo antes expuesto, al tratarse de materiales multimedia que apoyan el aprendizaje y fortalecimiento de las culturas y lenguas indígenas, 
se hace necesario adoptar una perspectiva teórica, pedagógica y lingüística, desde la cual se construya la propuesta didáctica y se aborde el diseño de situaciones de aprendizaje pertinentes (Casillas, 2012, p. 34).

Este panorama ya se ha vislumbrado en algunos centros educativos mediante la dotación o equipamiento de aulas de cómputo o aulas de medios, las cuales terminan siendo talleres de computación o de paquetería administrativa, con lo que se desvirtúa su propósito principal, que consiste en facilitar al personal docente las herramientas y recursos necesarios para fortalecer los procesos de enseñanza y aprendizaje a través de la tecnología, con el apoyo de programas multimedia pertinentes a los contextos indígenas.

Por lo tanto, hoy en día se deben producir materiales educativos multimedia con enfoques de diversidad lingüística y cultural, de tal manera que se vayan construyendo repositorios con este tipo de recursos que estén disponibles al momento que se requieran; es decir, habrá que planificar y crear materiales para el futuro. Para tal efecto, corresponde a maestras y maestros buscar espacios y mecanismos de capacitación y autoformación en el manejo de las TIC, con la finalidad de hacer un uso formativo de las mismas y colaborar en la construcción de plataformas interactivas con pertinencia cultural y lingüística. Respecto a la conectividad y equipamiento, corresponderá a la gestoría o a las políticas públicas que emanen del sistema educativo o de los diferentes niveles de gobierno.

\section{Referencias}

Alianza Nacional de Profesionales Indígenas Bilingües A. C. (ANPIBAC). (1980). Conclusiones de la mesa número 5 del I Seminario Nacional de Educación Bilingüe. En I Congreso Nacional "Los indígenas y su política educativa". Los Remedios, Ixmiquilpan, Hidalgo.
Hernández, F. G. (1979). De la educación indígena tradicional a la educación indígena bilingüe bicultural. Revista Mexicana de Ciencias Políticas y Sociales. México: Alianza Nacional de Profesionales Indígenas Bilingües (ANPIBAC), 50-63.

Hernández, L. R. (1982). México pluricultural, de la castellanización a la educación indígena bilingüe bicultural. México: Porrúa.

Jiménez, H. J. (2010). La telesecundaria en México: un breve recorrido histórico por sus datos y relatos. México: Dirección General de Materiales Educativos de la Subsecretaría de Educación Básica-SEP.

Nolasco, M. (1978). INI 30 años después, revisión crítica. México: Libros de México.

SEP-CONAFE-INI. (1981). Programa Nacional de Educación para todos. Informe anual de Labores 1980-81. México: Dirección General de Educación Indígena.

Villavicencio, Z. F. \& Salgado, A. E. (2011). Materiales multimedia en contextos de diversidad lingüística y cultural. México: CIESAS.

\section{Referencias electrónicas}

Aplicación (app) Yalam. Recuperado de https://play. google.com/store/apps/details?id=com.felix.seittu. yalam

Casillas, M. L. (2012). Retos de la expansión de la técnologias de la información y la comunicación en las comunidades indígenas de México. Revista Científica Electrónica de Educación y Comunicación en la Sociedad del Conocimiento, 1(12), 16-37. Recuperado de https://dialnet.unirioja.es/descarga/ articulo/3973021.pdf 
Escalante, C. (2013). Las cartillas de alfabetización de la campaña de 1944-1946 en México. Revista Mexicana de Historia de la Educación, I(1), 155-162. Recuperado de http://www.somehide.org/images/ documentos/articulosRevista1/somehide_1_7.pdf

Guerrero, M. \& Dote, F. (2011). Integración curricular de TIC's en la enseñanza de lenguas indígenas en Latinoamérica. Revista Electrónica Diálogos Educativos, 22, 15-31. Recuperado de https://www.google.com.mx/url?sa=t\&rct= $j \& q=\& e s r c=s \&$ source $=$ web \& $c d=1$ \&ved $=2 a h U$ KEwjSwai36-LiAhUS16wKHdOiA4YQFjAAegQ IABAC\&url=https\%3A\%2F\%2Fdialnet.unirioja. es $\% 2$ Fdescarga\%2Farticulo\%2F3931354. pdf\&usg=AOvVaw3dcsL-7ZYEwnGB3ZBJb1f7

López y Rivas, G. (1978). La castellanización de los indígenas para acelerar el etnocidio. Proceso. Recuperado de https://www.proceso.com. $\mathrm{mx} / 124553 /$ la-castellanizacion-de-los-indigenaspara-acelerar-el-etnocidio

Ortiz, G., Hernández, D. \& Romero, K. (2014). Educación, interculturalidad y tecnologías de la información y la comunicación. Hacia la construcción de un estado del arte en México. Comunicación y educación. Recuperado de http://congreso.pucp.edu. pe/alaic2014/wp-content/uploads/2013/09/GT4Ortiz-Hernandez-Romero.pdf

Sandoval, E. \& Mota, L. (2007). Indígenas y democracia en las tecnologías de información y comunicación (TICS). Recuperado de http://www.libertadciudadana. org/archivos/Biblioteca\%20Virtual/Documentos\%20 Informes $\% 20$ Indigenas/Documentos $\% 20$ Internacionales/Derecho/Indigenas\%20y\%20 Democracia\%20en\%20las\%20Tecnologias\%20 de\%20Informacion\%20y\%20Comunicacion.pdf

Software lingüístico-educativo denominado Xjuaní. Vocabulario ilustrado e interactivo en nueve lenguas indígenas de Oaxaca. Recuperado de http://www. cedelio.edu.mx/XJUANI/xjuani.html

Yagiits un material didáctico interactivo bilingüe (zapoteco-español). Recuperado de http://187.157.226.60/apps/tuleapp/ de Lenguas 


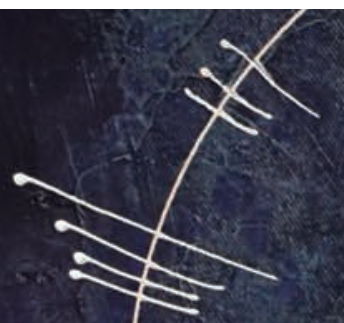

CP

el

(C)

(1)

1

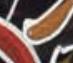

2

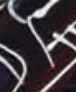

8

8

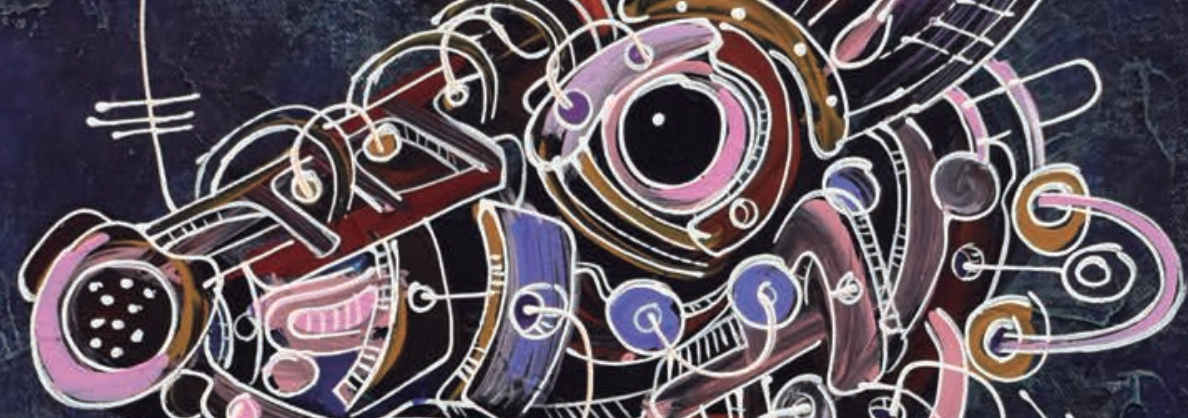

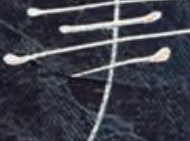
$\rightarrow$ का $\because \because 1$ base

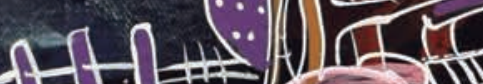

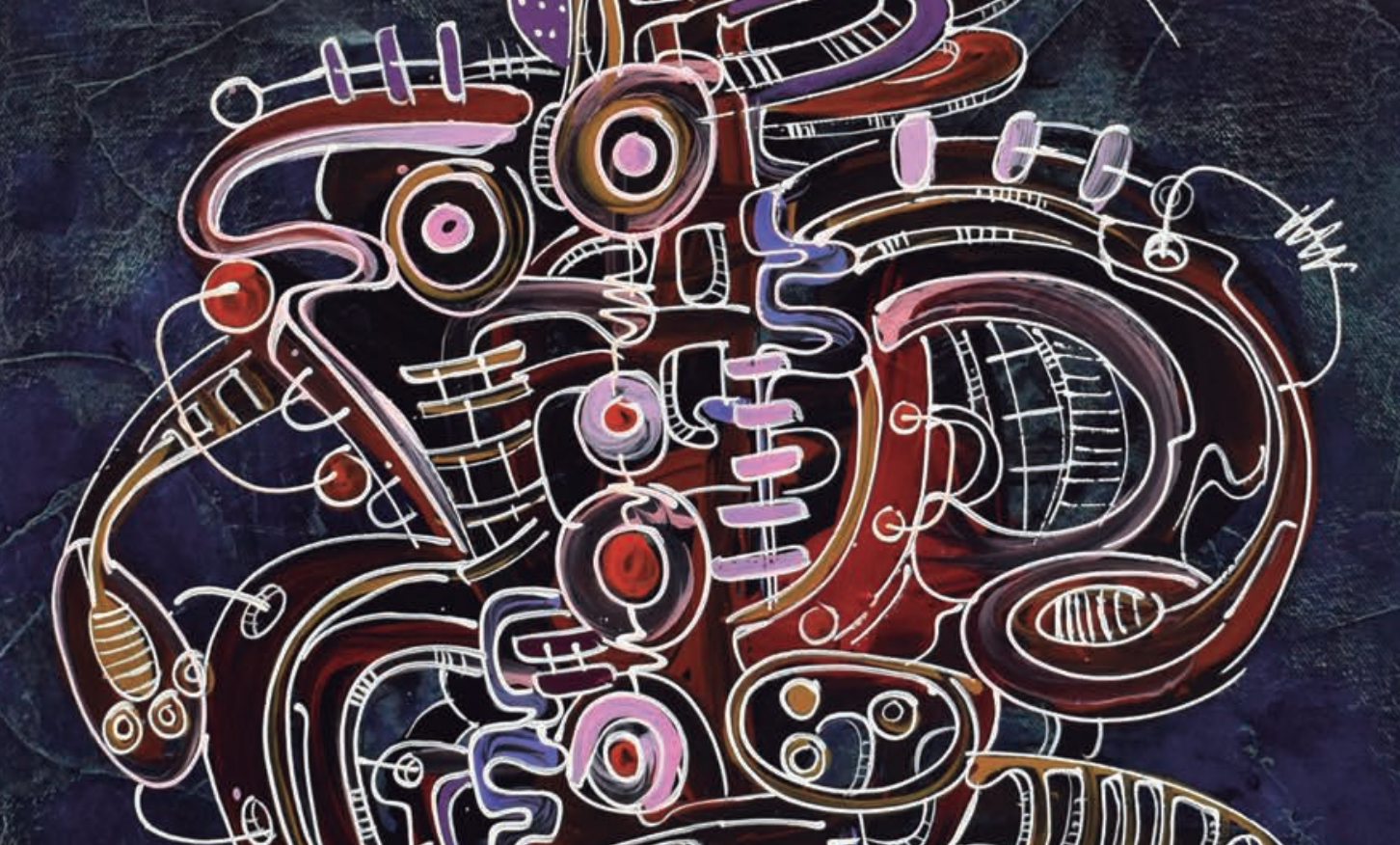
का $=4$ $\theta$ (2) $16(\sqrt{2}-3)=1$ 4 1 a $=$

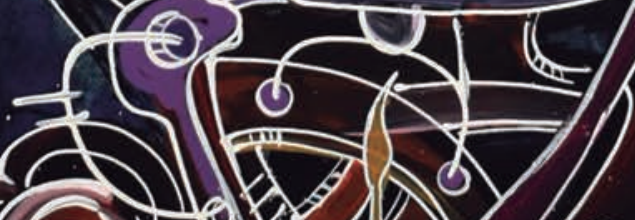

(2000 0 (1)
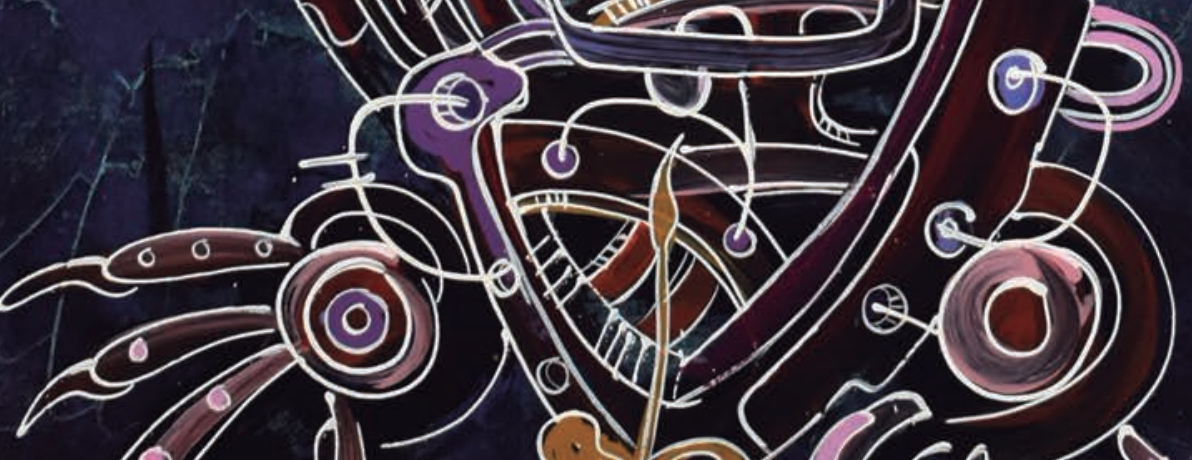

.

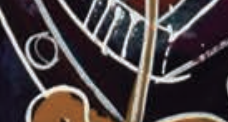




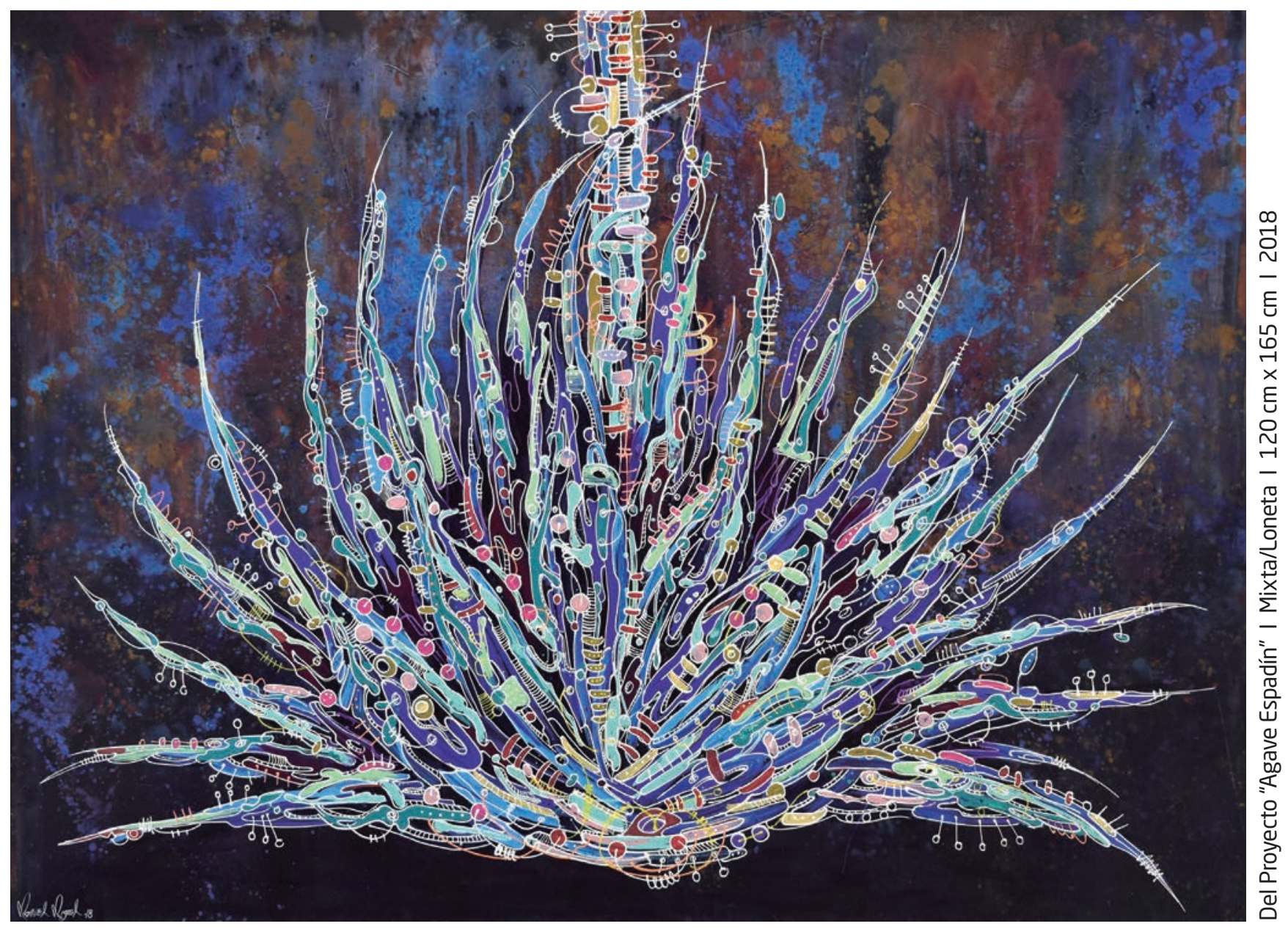

